\title{
IL RAPPORTO UOMO ACQUA NELLE SCELTE INSEDIATIVE E NELL'ORGANIZZAZIONE ANTROPICA DEL TERRITORIO: IL CONTRIBUto DElla CARTA ARCHEOlOgiCA DELla CAMPANIA
}

\section{THE RELATIONSHIP BETWEEN MAN AND WATER IN SETTLEMENT PATTERNS AND IN THE ORGANIZATION OF THE TERRITORY: THE CONTRIBUTION OF ARCHAEOLOGICAL MAP OF CAMPANIA}

\author{
GIONVANNA CERA \\ giovanna.cera@unisalento.it \\ UNIVERSITÀ DEL SALENTO ${ }^{1}$ \\ [RECIBIDO: 14/12/2017; ACEPTADO: 27/02/2018] \\ http://dx.doi.org/10.25267/Riparia.2018.v4.04
}

\section{RIASSUNTO}

Questo contributo analizza diversi aspetti relativi al rapporto tra uomo e acqua nelle aree interne della Campania settentrionale, in particolare nella media e alta valle del Volturno e nella Pianura Campana. Questa analisi, basata sui risultati delle indagini topografiche realizzate in questi territori negli ultimi anni (Carta Archeologica della Campania), ha lo scopo di illustrare lo sviluppo e le trasformazioni dell'insediamento in relazione alle zone parafluviali e alle vallate, in un periodo compreso tra l'epoca preistorica e quella romana. Si mettono inoltre in evidenza le forme di organizzazione territoriale connesse o condizionate dalla presenza di risorse idriche, nonché le dinamiche culturali ed economiche sviluppatesi in relazione ai principali fiumi e valli fluviali.

Parole CHIAve: Campania settentrionale; valli fluviali; risorse idriche; insediamento; organizzazione del territorio

1 Professore Associato. Topografia Antica. Dipartimento di Beni Culturali Edificio Corpo 2 - Via Dalmazio Birago, 64 - LECCE (LE) Ufficio, Secondo piano.

G. Cera, "Il rapporto uomo acqua nelle scelte insediative e nell'organizzazione antropica del territorio: il contributo della Carta Archeologica della Campania", RIPARIA 4 (2018), 90-110. 
RIPARIA VOL. 4 (2018)

\section{Abstract}

The following work analyzes several aspects concerning the relationship between man and water in the inner landscapes of Northern Campania, in particular in the Upper and Middle Volturno Valley and in the Capua Plain. This analisys is inspired by the results of the topographical surveys made in these territories in the last years (Archaeological Map of Campania). It aims to illustrate the development of the settlement in relation to the waterside zones, in a period between the Prehistory to the Roman Age. According to the available evidences about it, we can highlight some representative cases of forms of land use connected or conditioned by water recources. We can also focus the attention on the cultural and economic dynamics developed in relation to the main rivers and river valleys.

KEY WORDS: Northern Campania settentrionale; river valleys; water resources; settlement; land use. 
Le ricerche topografiche condotte nell'ambito del progetto dedicato alla Carta archeologica della Campania e intrapreso nell'ultimo quindicennio dalla Seconda Università di Napoli offrono uno sguardo di ampio respiro sulle caratteristiche del popolamento e sulle forme di organizzazione e di sfruttamento del territorio ${ }^{2}$. In questo contributo cercheremo di evidenziare, nello specifico, $i$ dati che emergono in relazione al rapporto tra uomo e risorse idriche, tra uomo e ambiente fluviale, nell'ambito del vasto orizzonte cronologico compreso tra l'epoca preistorica e quella romana.

Sono in particolare $i$ territori della Campania settentrionale interna (le regioni della media e alta valle del Volturno, del Calore e dei loro affluenti, la piana campana) quelli che consentono di enucleare i dati più interessanti in rapporto a questa tematica, in quanto gravitanti attorno ad alcuni tra $i$ principali corsi d'acqua della regione (fig. 1). I bassi terrazzi fluviali che caratterizzano la piana del Volturno (in particolare il suo alto e medio corso) presentano un tipo di habitat particolarmente adatto allo stanziamento di epoca preistorica, rappresentato non solo da terreni alluvionali adatti all'agricoltura intensiva o semi-intensiva, ma anche da condizioni ambientali favorevoli alla caccia e alla pesca e da facilità di approvvigionamento idrico ${ }^{3}$. Le aree più prossime al corso dei fiumi offrivano inoltre abbondanza di materia prima, selce e

\footnotetext{
2 Sullo "stato dell'arte" del progetto si vedano S. Quilici GIGLI, La Carta archeologica della Campania: l'impegno per la promozione di una coscienza culturale e civile, in Carta archeologica e ricerche in Campania, ATTA XV suppl., fasc. 4, 2010, 11-24; EADEM, Carta archeologica della Campania. Ricerche condotte e in corso per la promozione del territorio, in Carta archeologica e ricerche in Campania, ATTA XV suppl., fasc. 9, 2016, 7-15. ${ }^{3}$ È esemplificativo, in tal senso, il ritrovamento sporadico, nella zona della media valle del fiume (zona di Baia e Latina), di una scapola di elephans antiquus, ascrivibile al Paleolitico Inferiore: D. CAIAZZA, Archeologia e storia antica del mandamento di Pietramelara e del Montemaggiore, I, Preistoria ed età sannitica, Pietramelara 1986, 29; C. Albore Livadie, Testimonianze preistoriche nel territorio alifano-matesino, in L. Di Cosmo, A.M. VillucCi (a cura di), Il territorio alifano. Archeologia arte e storia. Atti del Convegno (S. Angelo d'Alife (CE) 1987), S. Angelo d'Alife (CE) 1990, 9.
}

"Il rapporto uomo acqua nelle scelte insediative e nell'organizzazione..." 
ciottoli, utili alla produzione di strumentario litico. La maggior parte dei giacimenti individuati lungo l'alta valle del fiume, ad esempio, documenta forme di frequentazione semi-stanziale o temporanea legate, oltre all'attività venatoria, soprattutto alla realizzazione di industria litica, considerata l'assoluta predominanza, in tali giacimenti, di residui di lavorazione (quali ciottoli e nuclei, con tracce di stacchi e non, pezzi amorfi battuti, e soprattutto schegge) rispetto agli oggetti finiti ${ }^{4}$.

Anche nel settore della media valle del fiume (in particolare il territorio cubulterino e quello alifano), nelle aree disposte lungo la destra e la sinistra idrografica del fiume, le evidenze documentate in base tanto a ricognizioni di superficie ${ }^{5}$ quanto a indagini stratigrafiche (come ad esempio l'agglomerato abitativo di località San Simeone, in vita tra l'Eneolitico e il Bronzo Antico $)^{6}$ rivelano, per la fase pre- e protostorica, una generalizzata situazione di scarsa conflittualità, suggerita dalla posizione degli insediamenti assai prossima al corso del Volturno, sui più bassi terrazzi fluviali della vallata o in corrispondenza di lievi rialzi morfologici, protetti dal pericolo di alluvioni e di acque stagnanti. Questa collocazione, poco favorevole al controllo del territorio, offriva tuttavia condizioni vantaggiose allo sfruttamento agricolo dei fertili terreni della piana e garantiva al

${ }^{4}$ G. CERA, Il territorio di Venafro, Carta archeologica e ricerche in Campania, ATTA XV suppl., fasc. V, Roma 2011, 131. Una situazione analoga è stata rilevata anche nei territori di Melizzano e Frasso Telesino, nel Beneventano, lungo la valle del Volturno e del Calore: A. SALERno, Lettura topografica dei dati archeologici dei territori di Melizzano e di Frasso Telesino, in Carta archeologica e ricerche in Campania, ATTA XV suppl., fasc. 10, 2017, 129-130.

5 G. CERA, Il territorio di Cubulteria, in Carta archeologica e ricerche in Campania, ATTA XV suppl., fasc. 1, 2004, 188, 190; S. MatalunA, Il territorio tra Allifae e Venafrum, in Carta archeologica e ricerche in Campania, ATTA XV suppl., fasc. 7, 2012, 115.

${ }^{6} \mathrm{P}$. TAlamo, Nuove scoperte di età eneolitica e del bronzo nell'ambito della preistoria del territorio alifano, in F. Miele, F. Sirano (a cura di), Ager allifanus. La Piana Alifana alla Luce delle Recenti Ricerche Archeologiche, Catalogo della Mostra (Alife, Museo Archeologico, 29 maggio - 29 ottobre 2004), Quaderni Campano-Sannitici IV, Piedimonte Matese (CE) 2004, $33-45$. 
contempo facilità di collegamento attraverso le rotte fluviali o gli itinerari di fondovalle.

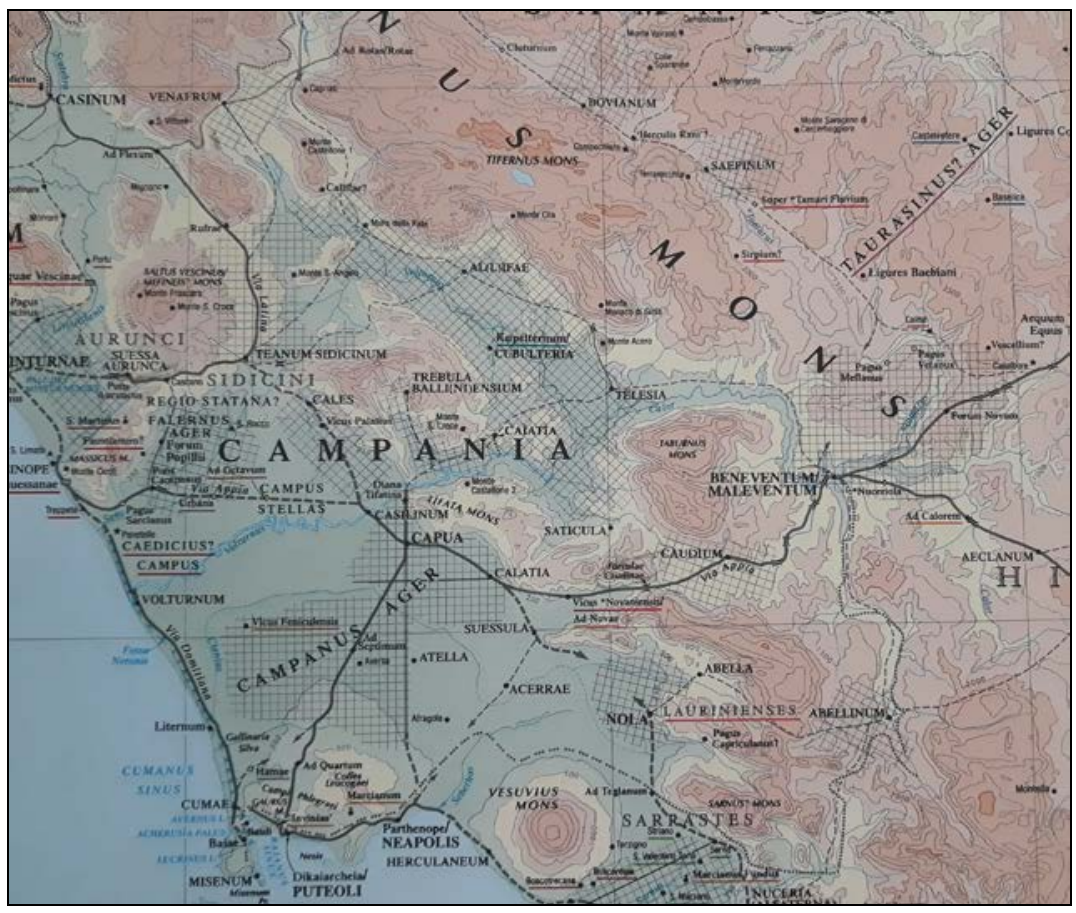

Fig. 1. Inquadramento geografico della Campania antica, con i principali centri, strade e fiumi menzionati nel testo (da R.J.A. TALBERT 2000)

Nel territorio telesino, più articolato dal punto di vista morfologico, la distribuzione dei contesti di questa stessa fase cronologica si pone invece per lo più in rapporto ai rilievi tufacei posti in prossimità della confluenza tra i fiumi Volturno e Titerno e tra Volturno e Calore (cammini naturali diretti verso le zone interne), anche se non mancano attestazioni in corrispondenza di piccoli dossi o di basse terrazze in prossimità dei fiumi ${ }^{7}$.

7 G. RENDA, Il territorio tra Monte Monaco e il fiume Calore. Lettura topografica dei dati archeologici, in Carta archeologica e ricerche in Campania, ATTA XV suppl., fasc. 4, 2010, 275-278 e fig. 258. Cfr. anche, più in generale, V. AmATO, A. SALERnO, Strategie

"Il rapporto uomo acqua nelle scelte insediative e nell'organizzazione..." 
In questo caso, più che allo sfruttamento di fertili suoli alluvionali, la collocazione in prossimità dei principali corsi d'acqua era ispirata soprattutto dalle prerogative viarie offerte dagli stessi, come ben documentato, ad esempio, lungo il percorso che collegava Alife e Venafro. Qui la presenza di due insediamenti, sorti sulle rive opposte del Volturno, a ridosso del punto di guado (area di Ponte Reale), denota l'attrattiva esercitata da questa zona di passaggio e di convergenza delle vie naturali di percorrenza terrestre e fluviale ${ }^{8}$.

Anche nel settore della pianura campana (territorio di Marcianise) emerge, per il Neolitico, l'Eneolitico e l'età del Bronzo, una netta tendenza delle scelte insediative verso zone non lontane dai principali corsi d'acqua, nello specifico l'antico fiume Clanis $^{9}$, sopravvissuto nell'opera di canalizzazione realizzata in età vicereale e noto con il nome di Regi Lagni ${ }^{10}$. In questa zona, nella fascia a sud del corso d'acqua, gli scavi archeologici hanno documentato opere di organizzazione territoriale e irreggimentazione idrica realizzate ai fini del migliore sfruttamento agricolo dei terreni (divisioni agrarie, canali) ${ }^{11}$.

di insediamento e paleoambienti nella Campania centro-settentrionale tra Neolitico ed Eneolitico, in Atti della XL Riunione Scientifica IIPP "Strategie di insediamento fra Lazio e Campania in età preistorica e protostorica" (Roma, Napoli, Pompei 2005), Firenze 2007, 127 145; C. Cesario, Tipologie insediamentali nell'area dell'alto casertano tra Eneolitico e Bronzo Antico, ibidem, 593-597.

8 Per la sponda venafrana: G. CERA, Il territorio di Venafro..., 132; per il settore alifano: C. Albore Livadie, R. La MotTa, Torcino (Capriati al Volturno, Prov. di Caserta), in Notiziario. Rivista di Scienze Preistoriche XLIV, 1-2, 1992, 242-243.

9 P. Fecondo, Il territorio di Marcianise, in Carta archeologica e ricerche in Campania, ATTA XV suppl., fasc. 8, 2014, 218-219.

10 G. Fiengo, I Regi Lagni e la bonifica della Campania felix durante il viceregno Spagnolo, Firenze 1988.

11 S. De Caro, in G. Franciosi (a cura di), Ager Campanus. Atti del Convegno Internazionale (Real Sito di S. Leucio 2001), Napoli 2002, 135-136. 


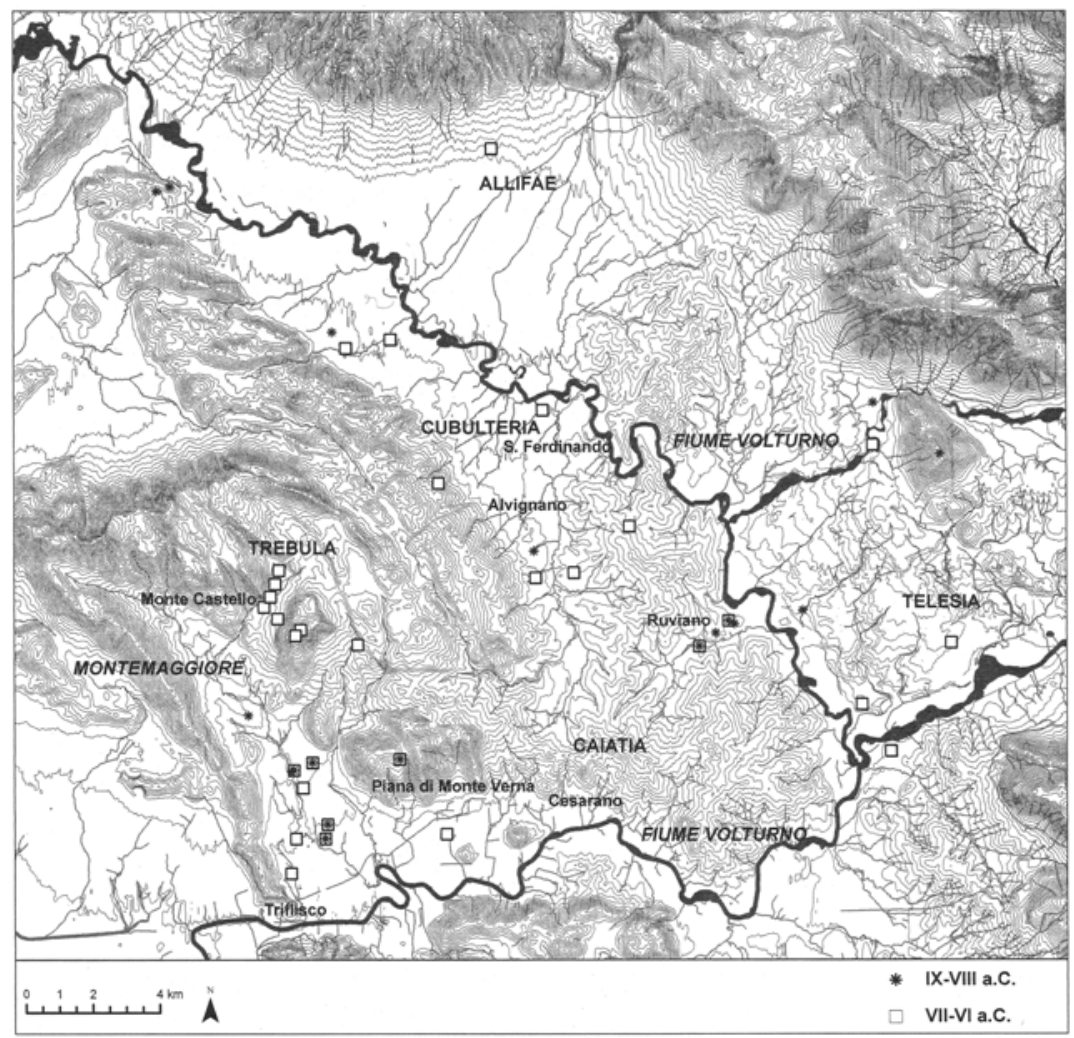

Fig. 2. Distribuzione dei principali nuclei insediativi nella media valle del Volturno tra il IX e il VI sec. a.C. (da P. CARFORA, ST. QUILICI GIGLI, G. RENDA, 2008)

Situazione analoga si riscontra nella piana a ovest di Capua, dove la distribuzione degli insediamenti appare fortemente condizionata dalla presenza del fiume Agnena - che in questo periodo costituiva la principale direttrice di traffico -, sia pure attraverso ben precise modalità di tutela dal pericolo delle alluvioni, come la collocazione in corrispondenza di dossi ${ }^{12} \mathrm{O}$

12 F. Guandalini, Il territorio a ovest di Capua, in Carta archeologica e ricerche in Campania, ATTA XV suppl., fasc. 2, 2004, 55-56.

"Il rapporto uomo acqua nelle scelte insediative e nell'organizzazione..." 
come anche l'apprestamento di palizzate o la realizzazione di canali di drenaggio ${ }^{13}$.

Anche in epoca arcaica la ricerca archeologica ha offerto testimonianze significative delle opere di regimazione idrica delle aree pianeggianti parafluviali, finalizzate al migliore rendimento agricolo dei suoli, oltre che a garantire le condizioni adeguate a una stabile occupazione degli insediamenti. Un lungo tratto di fossato con funzione di drenaggio, ascrivibile al VI-V sec. a.C., è stato messo in luce in un'area prossima all'alto corso del fiume Volturno, in località Acqua Solfa, nel comune di Pozzilli ${ }^{14}$, mentre molto più a sud, nell'agro di Acerra, è stata documentata una rete di canalizzazioni funzionali al deflusso delle acque superficiali di terreni a scarsa permeabilità, oltre che volti al controllo dei rischi causati dalle inondazioni del fiume Clanis ${ }^{15}$.

Infatti, dopo il periodo di scarsa attenzione per l'occupazione delle aree pianeggianti che caratterizza l'età del Bronzo (soprattutto la media età) - con la conseguente scelta di aree dominanti naturalmente protette ${ }^{16}$-, le modalità insediative

13 S. De CARo, Attività della Soprintendenza Archeologica di Napoli e Caserta nel 1998, in Atti Taranto XXXVIII, 1998, 640

14 A. Sarcina, Un sistema di drenaggio preromano a Pozzilli - Acqua Solfa, in Conoscenze 1-2, 2004, 43-44.

15 D. GiampaOla, Appunti per la storia del paesaggio agrario di Acerra, in Uomo acqua e paesaggio 1997, 226 ss.; S. DE CARO, L'attività archeologica a Napoli e Caserta nel 2002, in Atti Taranto XLII, 2002, 575-577. Una situazione analoga è attestata, per la stessa epoca, anche a Gricignano d'Aversa: E. LAForgiA, A. DE FilipPIS, Centuriazione a Gricignano d'Aversa, in G. Franciosi (a cura di), Ager Campanus..., 138; S. DE CARo, L'attività archeologica..., 577 s..

16 Per un'analisi delle scelte insediative attestate in Campania nel corso dell'antica e media età del Bronzo, cfr. C. Albore livadie, A. Cazzella, A. Marzocchella, M. PACCIARELLI, La struttura degli abitati del Bronzo antico e medio nelle Eolie e nell'Italia meridionale, in Atti della XXXV Riunione Scientifica IIPP "Le comunità della preistoria italiana. Studi e ricerche sul Neolitico e l'età del metalli” (Lipari 2000), I, Firenze 2003, 128-133; C. Albore Livadie, L'età del Bronzo antico e medio nella Campania nord-occidentale, in Atti della XL Riunione Scientifica IIPP "Strategie di insediamento fra Lazio e Campania in età preistorica e protostorica” (Roma, Napoli, Pompei 2005), Firenze 2007, 182 ss., 194-195. Nello specifico, per l'alta e media valle del Volturno: F. BAKER, K. Francis, P. HAYES, R. 
del periodo arcaico sono nuovamente contraddistinte da un generalizzato ritorno verso le valli fluviali (fig. 2$)^{17}$.

Questo nuovo assetto del popolamento, che mostra peraltro i segni di un deciso incremento, è senza dubbio legato a una più attenta e complessa organizzazione territoriale ai fini dello sfruttamento agricolo e risente al contempo di un generalizzato sviluppo economico, nell'ambito del quale lo smercio dei prodotti ha come fulcro la viabilità, anche quella fluviale.

La valle del Volturno costituisce, in tal senso, uno dei principali veicoli di diffusione di prodotti artigianali, nell'ambito degli intensi traffici commerciali intrattenuti da Capua con le regioni appenniniche interne, come dimostra la presenza di bucchero capuano nei principali centri della media e alta valle del

Hodges, J. Patterson, P. Roberts, The Field Surveys, in K. Bowes, K. Francis, R. Hodges (ed. by), Between Text and Territory. Survey and Excavations in the terra of San Vincenzo al Volturno (Archaeological Monographs of the British School at Rome), London 2006, 27; G. RENDA, Il territorio di Caiatia, in Carta archeologica e ricerche in Campania, ATTA XV suppl., fasc. 1, 2004, 397; C. CALASTRI, Il territorio di Trebula Balliensis, in Carta archeologica e ricerche in Campania, ATTA XV suppl., fasc. 3, 2006, 196-197; G. RENDA, Il territorio tra Monte Monaco ..., 278.

17 Per un quadro generale, cfr. G. Tagliamonte, I Sanniti, Milano 1997, 50 ss., $125-$ 126; P. Carfora, St. Quilici Gigli, G. Renda, Contributi della Carta archeologica della Campania: continuità e cambiamenti nel popolamento della Campania interna fra l'età arcaica e la fine delle guerre sannitiche, in Orizzonti IX, 2008, 127-129. Per settori specifici si rimanda, ad esempio, a P. TALAMO, L'area aurunca nel quadro dell'Italia centromeridionale. Testimonianze archeologiche di età arcaica (BAR Int.Ser. 384), Oxford 1987, in particolare 176 ss. per l'area aurunca; G. CERA, Il territorio di Venafro..., 135, 137 per il Venafrano e l'alta valle del Volturno; G. CERA, Il territorio di Cubulteria..., 191-195; per il territorio cubulterino; a G. RENDA Il territorio di Caiatia..., 398-399 per il territorio caiatino; C. CALAstRI, Il territorio di Trebula Balliensis..., 198-199 per il Trebulano; a F. SiRANo, Presenzano / Rufrae. Per una nuova immagine della piana nell'antichità, in D. Caiazza (a cura di), Presenzano e il Monte Cesima. Archeologia Arte e Storia di una Comunità, Atti del Convegno di Studi (Presenzano 2002), Piedimonte Matese (CE) 2002, 61-97; M.L. NAVA, F. SIRANO, Le fortificazioni megalitiche della media valle del Volturno nel contesto delle recenti scoperte archeologiche, in D. CAIAZZA (a cura di), Samnitice loqui. Studi in onore di Aldo L. Prosdocimi per il premio I Sanniti, II, Piedimonte Matese (CE) 2006, 272-274, per il comprensorio di Presenzano.

"Il rapporto uomo acqua nelle scelte insediative e nell'organizzazione..." 
fiume $^{18}$ (Alvignano $^{19}$, Alife $^{20}$, Presenzano ${ }^{21}$, Pozzillii $^{22}$, Colli al Volturno $^{23}$ ). Ed è sempre il Volturno a svolgere un ruolo importante nei collegamenti preferenziali tra la valle del Liri (Lazio meridionale), la Campania settentrionale e le regioni più interne del Sannio, favorendo la formazione di quella koiné culturale che viene convenzionalmente definita "civiltà della valle del Liri" 24 .

18 In generale, sulla diffusione del bucchero di Capua in altri contesti territoriali si rimanda a quanto esposto da M. MinOja, Il bucchero del Museo Provinciale Campano. Ricezione, produzione e commercio del bucchero a Capua, Pisa - Roma 2000, 118 ss. Sulla diffusione del bucchero attraverso le valli del Sacco, del Liri e del Volturno, P. FORTINI, Rinvenimento di buccheri nei territori compresi tra il Liri ed il Volturno, in QuadAEI IV, 1981, 91-93.

19 G. CERA, Il territorio di Cubulteria..., 117, 143, 192, 194. Anche l'antefissa arcaica, raffigurante Artemis con due volatili ai lati, proveniente da Alvignano (EADEM, p. 144 n. 116) sembra riconducibile, per caratteristiche tematiche e formali, a serie capuane di età arcaica (C. RESCIGNO, Tetti campani, Roma 1998, 348-349, in particolare 349).

20 M. Lista, C. Ziviello, Necropoli di Alife, in Annali della Scuola Superiore Normale di Pisa, s. III, XXI, 1, 1991, 32; G. TAgLiamonte, L'età preromana e sannitica. Le necropoli preromane nell'ambito della cultura sannitica nel territorio alifano, in $\mathrm{F}$. Miele, F. Sirano (a cura di), Ager allifanus..., 53-54.

21 W. Johannowsky, Presenzano: necropoli in località Robbia, in Studi sull'talia dei Sanniti, Roma 2000, 17 e 18 nota 10.

22 S. Capini, La necropoli di Pozzilli, in Sannio. Pentri e Frentani dal VI al I sec. a.C., Roma 1980, 112-128; S. CAPINI, L'alta valle del Volturno: la necropoli di Pozzilli, in S. CAPINI, A. Di Niro (a cura di), Samnium: archeologia del Molise, Roma 1991, 57-59.

23 S. CAPINI, L'insediamento di Monte San Paolo a Volturno e la guerra nel Sannio nel 293 a.C., in $B d A$ 16-18, 1992, 36; M. RADDI, Il commercio del bucchero nell'alta valle del Volturno attraverso i reperti di Colli a Volturno ed il suo territorio, in Annuario Associazione Storica del Medio Volturno 2000, 249-264.

24 W. Johannowsky, Materiali di età arcaica dalla Campania, Napoli 1983, 289-294. Si vedano anche P. TAlamo, L'area aurunca..., 163 ss.; G. TAgliamonte, I Sanniti.., 71 ss.; G. Gasperetti, C. Passaro, S. De Caro, Novità dal territorio degli Ausoni, in M. Barra Bagnasco, E. De Miro, A. Pizzone (a cura di), Origine e incontri di culture nell'antichità. Magna Grecia e Sicilia. Stato degli studi e prospettive di ricerca. Atti dell'incontro di Studi (Messina 1996), Catanzaro 1999, 145-146; F. SIRANO, Identità culturali nella Campania settentrionale: un aggiornamento, in C. CoRsi, E. POLITO (a cura di), Dalle sorgenti alla foce. Il bacino del Liri - Garigliano nell'antichità: culture contatti scambi, Atti del Convegno (Frosinone - Formia 2005), Roma 2008, 46 ss. Cfr. anche G. TAgliamonTe, L'età preromana e sannitica..., 52-53; F. SiRANO, Presenzano-Rufrae. Nuovi dati per la storia del popolamento nella Campania settentrionale tra età arcaica e classica sullo sfondo delle dinamiche di lungo periodo, in P. AtTema, A. NijBoer, A. Zifferero (ed. by), Papers in Italian Archaeology VT. Communities and Settlements from the Neolithic to the 
Per l'epoca alto- e mediorepubblicana (V - IV sec. a.C.) l'aspetto che maggiormente caratterizza il popolamento di questi territori è senza dubbio quello legato allo sviluppo delle fortificazioni d'altura. Oltre alla ovvia posizione dominante, la scelta dei luoghi per l'occupazione privilegia la possibilità di collegamento reciproco e le prerogative di controllo della viabilità. Il rapporto con la rete idrica del territorio acquista dunque in alcuni casi particolare importanza, ai fini dello sbarramento dei più facili accessi attraverso le vallate fluviali. Questo tipo di situazione appare ben documentata, ad esempio, nel territorio telesino, dove gli apprestamenti difensivi di altura noti in località San Pasquale e Monte Acero si pongono a controllo della valle del Titerno, mentre quelli di località La Rocca e Monte Pugliano guardano gli accessi attraverso il fiume Calore e la valle telesina ${ }^{25}$. Questo complesso sistema posto a guardia del territorio e a diretto controllo delle principali direttrici viarie si amplifica grazie alla realizzazione di un'ulteriore serie di fortificazioni (per lo più di piccole dimensioni) poste sull'altro versante dello stesso fiume Calore, tutte in collegamento visivo tra di loro e con i siti fortificati dell'area telesina (fig. 3$)^{26}$. Situazione simile si riscontra anche nell'Alifano, dove gli apprestamenti d'altura di Mandra Castellone, di Capriati al Volturno, di Monte Castellone a Ciorlano e di Roccavecchia di Pratella sono disposti a sbarramento degli accessi sul fiume Volturno ${ }^{27}$.

Early Medieval Period. Proceedings of the 6th Conference of Italian Archaeology (Groningen 2003) (BAR Int.Ser.1452), I, Oxford 2005, 305, 309.

25 G. RendA, Il territorio tra Monte Monaco..., 284-286.

26 S. Quilici GigLi, Lettura storica dei dati archeologici: dall'epoca sannitica all'epoca imperiale, in Carta archeologica e ricerche in Campania, ATTA XV suppl., fasc. 10, 2017, 137-138.

27 S.P. OAKLeY, The Hill-forts of the Samnites, London 1995, 36-38; S. MATALunA, Il territorio tra Allifae e Venafrum, in Carta archeologica e ricerche in Campania, ATTA XV suppl., fasc. 7, 2012, 118.

"Il rapporto uomo acqua nelle scelte insediative e nell'organizzazione..." 


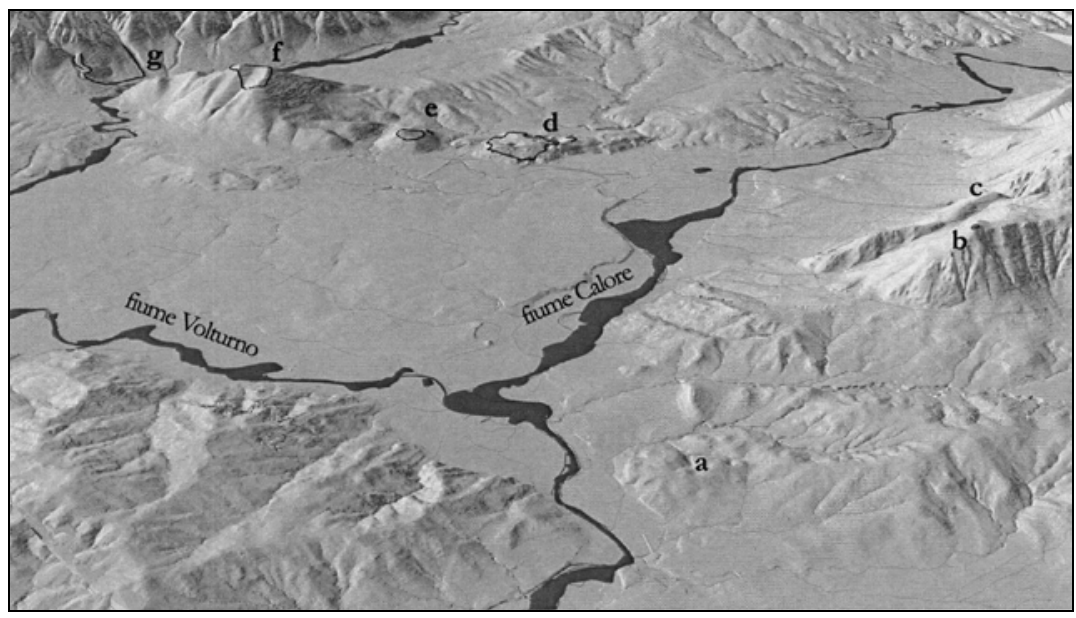

Fig. 3. Siti fortificati sannitici nell'area telesina e nella valle del Calore (da S. QUILICI GIGLI, 2017)

Forme di sfruttamento delle risorse idriche rivolte all'approvvigionamento idrico degli insediamenti rurali e al potenziamento dell'attività agricola sono rappresentate da complessi sistemi di canalizzazione e di raccolta o irreggimentazione idrica, scavati nel banco tufaceo, spesso di difficile inquadramento cronologico, sia pure da ascrivere genericamente al periodo medio- e tardorepubblicano. Ci si riferisce, ad esempio, agli apprestamenti documentati in territorio caiatino $^{28}$, così come in quello trebulano e attorno alla colonia di Cales $^{29}$, alcuni dei quali captavano le acque sorgive del Montemaggiore, per poi incanalarle verso gli insediamenti della pianura; altri invece svolgevano una funzione di drenaggio dei suoli.

\footnotetext{
${ }^{28}$ G. RENDA, Il territorio di Caiatia..., 352-353 n. 362, 407.

${ }^{29}$ Cfr. K. ØDEgARD, Drainage and Colonization: the case of Cales, in Uomo, acqua e paesaggio 1997, 213-224; C. CAlastri, Il territorio di Trebula Balliensis..., 223. Cfr. anche G. Gasperetti, A. Balasco, L.M. Proietti, L. Crimaco, Testimonianze archeologiche delle infrastrutture idrauliche di età romana tra il Garigliano e il Massico, in Uomo, acqua e paesaggio..., 1997, 239-262.
} 
In epoca tardorepubblicana, in concomitanza con una sempre più capillare diffusione del popolamento nelle campagne, si assiste a un potenziamento dei sistemi di raccolta e approvvigionamento idrico, a servizio di ville, impianti rurali e produttivi, i più importanti dei quali erano direttamente alimentati dagli acquedotti. Nell'esiguità della documentazione archeologica, spiccano i dati relativi alle diramazioni collegate all'acquedotto augusteo di Venafro ${ }^{30}$, alle quali fa peraltro esplicito riferimento il testo del noto editto relativo alla gestione e all'amministrazione dell'opera; in esso vengono enunciate le condizioni da rispettare nella realizzazione di canali di derivazione, da collocare possibilmente sotto terra, in corrispondenza o a fianco di una via pubblica $^{31}$. Allacciato alle sorgenti del Volturno, l'acquedotto procedeva lungo le propaggini delle montagne poste alle spalle di Santa Maria Oliveto, Pozzilli e Venafro, con un percorso in gran parte sotterraneo, e riforniva, attraverso alcuni bracci secondari, un'estesa villa romana messa in luce (e nota grazie alla documentazione d'archivio) in località Pozzo Sfondato ${ }^{32}$ e un complesso termale pubblico posto sulla riva del Volturno, in località Acqua Solfa ${ }^{33}$.

\footnotetext{
30 F. Frediani, "L'acquedotto augusteo" di Venafro, in Campania romana. Studi e Materiali, I, Napoli, 1938, 173- 185; W. Bowden, The Venafro Aqueduct, in K. Boews, K. Francis, R. Hodges (ed. by), Between Text and Territory. Survey and Excavations in the Terra of San Vincenzo al Volturno, London, 2006, 313-318.

31 (... neve / eae fistulae aut rivos nisi sub terra, quae terra itineris viae publicae limi / tisve erit ponantur conlocentur: CIL X, 4842, rr. 45-46 ss.). Sull'editto dell'acquedotto venafrano: CIL X, 4842 = ILS 5743; A. PANTONI, L'editto angusteo di Venafro e una sua replica alle fonti del Volturno, in RendPontAcc 33, 1960-61, 155-171; P. ZANOVELLO, Le fonti epigrafiche, in I. RIERA (a cura di), Utilitas necessaria. Sistemi idraulici nell'Italia romana, Milano 1994, 130 ss. 32 G. CERA, Contributi alla conoscenza del territorio di Venafro in epoca romana, in Orizzonti VIII, 2007 [2008], 43-55.

33 L. SCARoina, Il complesso termale dell'Acqua Solfa a Pozzilli, in Conoscenze 1-2, 2004, 27 ss. e figg. 1 A, $2-5$.
}

"Il rapporto uomo acqua nelle scelte insediative e nell'organizzazione..." 


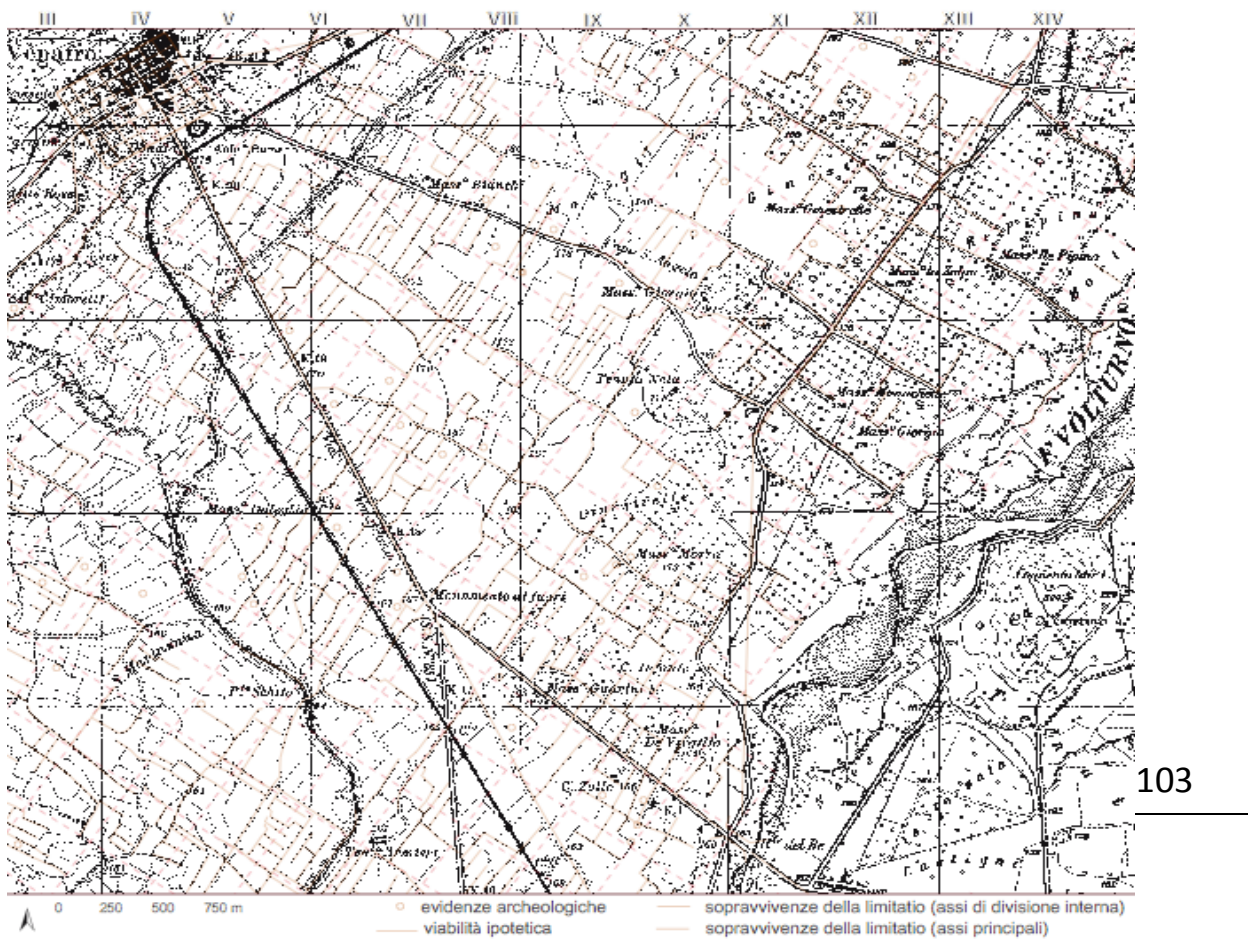

Fig. 4. La divisione agraria dell'ager venafranus (particolare del settore compreso tra Venafro e il fiume Volturno, da G. CERA, 2011)

L'utilizzo dell'acqua a fini produttivi è poi ben esemplificata, sempre nel territorio venafrano, dalla singolare testimonianza relativa all'esistenza di una sorta di mulino ad acqua; questo, posto sulla riva del Torrente Tuliverno (non lontano dall'attuale centro di Pozzilli), sfruttava la forza della corrente per azionare le pale della ruota e avviare di conseguenza la rotazione della macina per la produzione di farina ${ }^{34}$.

\footnotetext{
${ }^{34}$ Durante lavori di sistemazione dell'alveo del torrente, sulla sponda del corso d'acqua, furono individuati nel 1908 alcuni particolari segni di una ruota lignea, da cui fu possibile ricostruire il dispositivo originario: si trattava di un mulino dotato di una ruota idraulica a pinne (0,74 $\mathrm{m}$ di diametro), del tipo descritto da Vitruvio (VITR. 10.5), che azionava due mole circolari in pietra vulcanica di Roccamonfina (diam. 0,80 m, spess.
} 
Fiumi e sorgenti rappresentavano per il territorio elementi di indubbio vantaggio (e in quanto tali spesso sacralizzati) ${ }^{35}$, ma talvolta anche di pericolo: straripamenti e inondazioni non dovevano essere infrequenti. Non a caso le fonti letterarie ricordano il carattere violento e imprevedibile del Volturno, descrivendone l'impeto della corrente ${ }^{36}$ (significativi gli appellativi celer $^{37}$ e rapax $^{38}$ ), anche se la poetica immagine di Stazio, pur rappresentando il carattere minaccioso del fiume, ricorda come le opere di irreggimentazione delle sponde attuate nella zona di foce abbiano contribuito a diminuire notevolmente l'incidenza rovinosa di tali fenomeni ${ }^{39}$.

In tal senso i regolari sistemi di suddivisione agraria del terreno attuati dai Romani su vaste porzioni di territorio conquistato (o comunque acquisito) costituiscono una valida forma di organizzazione del paesaggio e un metodo efficace di gestione e di controllo delle acque. Lo studio dei numerosi impianti centuriali che caratterizzano le pianure fluviali dei

$0,25 \mathrm{~m}$ circa) rinvenute nelle vicinanze. L. JACONO, La ruota idraulica romana di Venafro, in L'Ingegnere 12-15, 1938, XVII, 1-4 (dell'estratto); V. MARCHIS, G. SCALVA, La scienza e le tecniche dell'acqua, in A. Ciarallo, E. De Carolis (a cura di), Homo faber. Natura, scienza e tecnica nell'antica Pompei, Milano, 1999, 291-293, p. 293; anche 325 scheda n. 417.

${ }^{35}$ Numerosi, nel comprensorio della Campania settentrionale, i luoghi di culto connessi a fiumi o a sorgenti e riferibili nella maggior parte dei casi a epoca preromana. Si tratta per lo più di santuari di modesta entità, probabilmente all'aperto o comunque poco monumentali, che costituivano punti di aggregazione della comunità rurale. Cfr. ad esempio L. CerChiaI, Acque, grotte e dei: i santuari indigeni nell'Italia meridionale, in Ocnus 7, 1999, 205-207, 217; P. CARAFA, Culti e santuari della Campania antica, Roma 2008, passim; F. Miele, Aree sacre connesse a culti di divinità femminili e maschili presso fonti, sorgenti e punti di guado nella media valle del fiume Volturno, in H. DI Giuseppe, M. SERLORENZI (a cura di), I riti del costruire nelle acque violate, Roma 2010, 209-244; G. Tagliamonte, I culti preromani, in S. Capini, P. Curci, M.R. Picuti (a cura di), Fana, templa, delubra. Corpus dei luoghi di culto dell'Italia antica, 3, Regio IV, Alife, Bojano, Sepino, Roma 2014, 11-14.

${ }^{36}$ Cfr. ad esempio Ov. Met. 15.714.

37 LUCAN. 2.423.

38 Claud. Panegyris in Consulatu Probii et Olybrii fratrum 256.

${ }^{39}$ STAT. Silv. 4.3.67 ss.

"Il rapporto uomo acqua nelle scelte insediative e nell'organizzazione..." 
comprensori presi in esame mette in evidenza lo stretto legame tra limitatio e assetto geomorfologico e idrografico. Nel territorio cubulterino e in quello venafrano, ad esempio, appare evidente come gli assi catastali di maggiore importanza coincidessero per lo più con fossi di drenaggio o con canali di irreggimentazione dei piccoli corsi d'acqua (fig. 4); essi muovono in direzione del Volturno, assecondando il decremento altimetrico del suolo e rappresentando quindi un elemento importante nella sistemazione territoriale, grazie alla loro funzione di bonifica e di smaltimento delle acque superficiali verso il grande collettore fluviale $^{40}$.

Molto meno riconoscibili e conservate sono invece le testimonianze archeologiche relative a opere di arginatura delle sponde fluviali. Un unico esempio è stato rilevato nel territorio cubulterino, in località Ponte del comune di Baia e Latina, lungo il medio corso del Volturno. Qui, oltre alla presenza di resti di un ponte (forse ascrivibile a età tardo-repubblicana o augustea), si conservano lunghi tratti di muri d'argine e di probabili banchine, realizzati su entrambe le sponde del fiume, a ridosso del ponte stesso, allo scopo di difendere le rive dalla forza erosiva della corrente e al fine di regolarizzarne la portata. Si osservano inoltre, addossati ai muri d'argine, i resti di strette scalette, evidentemente utili a operazioni di sbarco e imbarco di merci, i quali parrebbero suggerire una funzione di tale apprestamento anche come scalo fluviale $^{41}$.

${ }^{40}$ G. CERA, Il territorio di Cubulteria..., 221-222; G. CERA, Il territorio di Venafro..., 162. Cfr. anche, per altri comprensori, F. GUANDALINI, Il territorio a ovest..., pp. 5859; G. Tagliamonte, F. Miele, L'ager allifanus, in Ager Campanus...., 191-199. Cfr. in generale, per la piana campana, M. MonACO, Centuriazione e gestione delle acque. Considerazioni in tema di aspetti agrari nell'ager campanus, in G. FRANCIOSI (a cura di), La romanizzazione della Campania antica, 1, Napoli 2002, 87-123.

${ }^{41}$ G. CERA, Il territorio di Cubulteria..., 60-63 sito 22. 


\section{Bibliografia}

C. Albore Livadie, Testimonianze preistoriche nel territorio alifanomatesino, in L. Di Cosmo, A.M. VILLUCCI (a cura di), Il territorio alifano. Archeologia arte e storia. Atti del Convegno (S. Angelo d'Alife (CE) 1987), S. Angelo d'Alife (CE) 1990, 7-23.

C. Albore Livadie, R. La MotTA, Torcino (Capriati al Volturno, Prov. di Caserta), in Notiziario. Rivista di Scienze Preistoriche XLIV, 1-2, 1992, 242-243.

C. Albore livadie, A. Cazzella, A. Marzocchella, M. PACCIARELLI, La struttura degli abitati del Bronzo antico e medio nelle Eolie e nell'Italia meridionale, in Atti della XXXV Riunione Scientifica IIPP "Le comunità della preistoria italiana. Studi e ricerche sul Neolitico e l'età del metalli” (Lipari 2000), I, Firenze 2003, 113-142.

C. Albore Livadie, L'età del Bronzo antico e medio nella Campania nord-occidentale, in Atti della XL Riunione Scientifica IIPP "Strategie di insediamento fra Lazio e Campania in età preistorica e protostorica" (Roma, Napoli, Pompei 2005), Firenze 2007, 179-203.

V. Amato, A. SALERnO, Strategie di insediamento e paleoambienti

nella Campania centro-settentrionale tra Neolitico ed Eneolitico, in Atti della XL Riunione Scientifica IIPP "Strategie di insediamento fra Lazio e Campania in età preistorica e protostorica" (Roma, Napoli, Pompei 2005), Firenze 2007, 127-145.

F. Baker, K. Francis, P. Hayes, R. Hodges, J. Patterson, P. Roberts, The Field Surveys, in K. BOwes, K. Francis, R. HODGES (ed. by), Between Text and Territory. Survey and Excavations in the terra of San Vincenzo al Volturno (Archaeological Monographs of the British School at Rome), London 2006.

W. Bowden, The Venafro Aqueduct, in K. Boews, K. Francis, R. Hodges (ed. by), Between Text and Territory. Survey and Excavations in the Terra of San Vincenzo al Volturno, London, 2006, 313-318.

D. CAIAZZA, Archeologia e storia antica del mandamento di Pietramelara e del Montemaggiore, I, Preistoria ed età sannitica, Pietramelara 1986.

C. CALASTRI, Il territorio di Trebula Balliensis, in Carta archeologica e ricerche in Campania, ATTA XV suppl., fasc. 3, 2006, 11-228.

S. CAPINI, La necropoli di Pozzilli, in Sannio. Pentri e Frentani dal VI al I sec. a.C., Roma 1980, 112-128.

"Il rapporto uomo acqua nelle scelte insediative e nell'organizzazione..." 
S. CAPINI, L'alta valle del Volturno: la necropoli di Pozzilli, in S. CAPInI, A. Di Niro (a cura di), Samnium: archeologia del Molise, Roma 1991, 57-59.

S. CAPINI, L'insediamento di Monte San Paolo a Volturno e la guerra nel Sannio nel 293 a.C., in BdA 16-18, 1992, 33-42.

P. CARAFA, Culti e santuari della Campania antica, Roma 2008.

P. CArfora, ST. Quilici Gigli, G. RendA, Contributi della Carta archeologica della Campania: continuità e cambiamenti nel popolamento della Campania interna fra l'età arcaica e la fine delle guerre sannitiche, in Orizzonti IX, 2008, 127-133.

G. CERA, Il territorio di Cubulteria, in Carta archeologica e ricerche in Campania, ATTA XV suppl., fasc. 1, 2004, 19-235.

G. CERA, Contributi alla conoscenza del territorio di Venafro in epoca romana, in Orizzonti VIII, 2007 [2008], 43-55.

G. CERA, Il territorio di Venafro, Carta archeologica e ricerche in Campania, ATTA XV suppl., fasc. V, Roma 2011.

L. CerchiaI, Acque, grotte e dei: i santuari indigeni nell'Italia meridionale, in Ocnus 7, 1999, 205-222.

C. CESARIO, Tipologie insediamentali nell'area dell'alto casertano tra Eneolitico e Bronzo Antico, ibidem, 593-597.

S. De CAro, Attività della Soprintendenza Archeologica di Napoli e Caserta nel 1998, in Atti Taranto XXXVIII, 1998, 633-661.

S. DE CARO, L'attività archeologica a Napoli e Caserta nel 2002, in Atti Taranto XLII, 2002, 569-621.

S. De Caro, in G. Franciosi (a cura di), Ager Campanus. Atti del Convegno Internazionale (Real Sito di S. Leucio 2001), Napoli 2002.

P. FECONDO, Il territorio di Marcianise, in Carta archeologica e ricerche in Campania, ATTA XV suppl., fasc. 8, 2014, 111-223.

G. FIEngO, I Regi Lagni e la bonifica della Campania felix durante il viceregno Spagnolo, Firenze 1988.

P. FORTINI, Rinvenimento di buccheri nei territori compresi tra il Liri ed il Volturno, in QuadAEI IV, 1981, 91-93.

F. FrEDIANI, "L'acquedotto augusteo" di Venafro, in Campania romana. Studi e Materiali, I, Napoli, 1938, 173- 185.

G. Gasperetti, A. Balasco, L.M. Proietti, L. Crimaco, Testimonianze archeologiche delle infrastrutture idrauliche di età romana tra il Garigliano e il Massico, in Uomo, acqua e paesaggio, Atti 
dell'incontro sul tema Irreggimentazione delle acque e trasformazione del paesaggio antico (S. Maria Capua Vetere 1996), in ATTA II suppl., 1997, 239-262.

G. Gasperetti, C. PAssaro, S. DE CARO, Novità dal territorio degli Ausoni, in M. BArra BAgnasco, E. DE Miro, A. PizZONE (a cura di), Origine e incontri di culture nell'antichità. Magna Grecia e Sicilia. Stato degli studi e prospettive di ricerca. Atti dellincontro di Studi (Messina 1996), Catanzaro 1999, 145-158.

D. GiAmpaOLA, Appunti per la storia del paesaggio agrario di Acerra, in Uomo acqua e paesaggio 1997,225-238.

F. GUANDALINI, Il territorio a ovest di Capua, in Carta archeologica e ricerche in Campania, ATT A XV suppl., fasc. 2, 2004, 11-66.

L. JACONO, La ruota idraulica romana di Venafro, in L'Ingegnere 12-15, 1938, XVII, 1-4 (dell'estratto).

W. JOHANNOWSKY, Materiali di età arcaica dalla Campania, Napoli 1983.

W. JOHANNOWSKY, Presenzano: necropoli in località Robbia, in Studi sull'Italia dei Sanniti, Roma 2000, 16-19.

E. LAFOrgiA, A. DE FilipPis, Centuriazione a Gricignano d'Aversa, G. FRAnCIOSI (a cura di), Ager Campanus. Atti del Convegno Internazionale (Real Sito di S. Leucio 2001), Napoli 2002, 137-164.

M. Lista, C. Ziviello, Necropoli di Alife, in Annali della Scuola Superiore Normale di Pisa, s. III, XXI, 1, 1991, 29-60.

V. Marchis, G. ScAlVA, La scienza e le tecniche dell'acqua, in A. Ciarallo, E. DE CAROLIS (a cura di), Homo faber. Natura, scienza e tecnica nell'antica Pompei, Milano, 1999, 291-293.

S. MATALUNA, Il territorio tra Allifae e Venafrum, in Carta archeologica e ricerche in Campania, ATT A XV suppl., fasc. 7, 2012, 114-128.

F. MIELE, Aree sacre connesse a culti di divinità femminili e maschili presso fonti, sorgenti e punti di guado nella media valle del fiume Volturno, in H. DI GIUSEPPE, M. SERLORENZI (a cura di), I riti del costruire nelle acque violate, Roma 2010, 209-244.

M. MinojA, Il bucchero del Museo Provinciale Campano. Ricezione, produzione e commercio del bucchero a Capua, Pisa - Roma 2000.

M. MONACO, Centuriazione e gestione delle acque. Considerazioni in tema di aspetti agrari nell'ager campanus, in G. FRANCIOSI (a cura di), La romanizzazione della Campania antica, 1, Napoli 2002, 87-123.

M.L. NAVA, F. SIRANO, Le fortificazioni megalitiche della media valle del Volturno nel contesto delle recenti scoperte archeologiche, in D. CAIAZZA (a cura di), Samnitice loqui. Studi in onore di Aldo L. Prosdocimi per il premio I Sanniti, II, Piedimonte Matese (CE) 2006, 269-288.

"Il rapporto uomo acqua nelle scelte insediative e nell'organizzazione..." 
S.P. OAKLEY, The Hill-forts of the Samnites, London 1995.

K. ØDEGARD, Drainage and Colonization: the case of Cales, in Uomo, acqua e paesaggio, Atti dell'incontro sul tema Irreggimentazione delle acque e trasformazione del paesaggio antico (S. Maria Capua Vetere 1996), in ATTA II suppl., 1997, pp. 213-224.

A. PANTONI, L'editto augusteo di Venafro e una sua replica alle fonti del Volturno, in RendPontAcc 33, 1960-61, 155-171.

S. QUILICI GIGLI, La Carta archeologica della Campania: l'impegno per la promozione di una coscienza culturale e civile, in Carta archeologica e ricerche in Campania, ATTA XV suppl., fasc. 4, 2010, 11-24.

S. Quilici Gigli, Lettura storica dei dati archeologici: dall'epoca sannitica all'epoca imperiale, in Carta archeologica e ricerche in Campania, ATTA XV suppl., fasc. 10, 2017,135-154.

S. Quilici GIGLI, Carta archeologica della Campania. Ricerche condotte e in corso per la promozione del territorio, in Carta archeologica e ricerche in Campania, ATTA XV suppl., fasc. 9, 2016, 7-15.

M. RADDI, Il commercio del bucchero nell'alta valle del Volturno attraverso i reperti di Colli a Volturno ed il suo territorio, in Annuario Associazione Storica del Medio Volturno 2000, 249-264.

G. RENDA, Il territorio di Caiatia, in Carta archeologica e ricerche in Campania, ATT A XV suppl., fasc. 1, 2004, pp. 239-423.

G. RENDA, Il territorio tra Monte Monaco e il fiume Calore. Lettura topografica dei dati archeologici, in Carta archeologica e ricerche in Campania, ATTA XV suppl., fasc. 4, 2010, 275-311.

C. RESCignO, Tetti campani, Roma 1998.

A. SALERNO, Lettura topografica dei dati archeologici dei territori di Melizzano e di Frasso Telesino, in Carta archeologica e ricerche in Campania, ATTA XV suppl., fasc. 10, 2017, 129-133.

A. SARCINA, Un sistema di drenaggio preromano a Pozzilli - Acqua Solfa, in Conoscenze 1-2, 2004, 43-44.

L. SCAroina, Il complesso termale dell'Acqua Solfa a Pozzilli, in Conoscenze 1-2, 2004, 27-36.

F. SiRANO, Presenzano / Rufrae. Per una nuova immagine della piana nell'antichità, in D. CAIAZZA (a cura di), Presenzano e il Monte Cesima. Archeologia Arte e Storia di una Comunità, Atti del Convegno di Studi (Presenzano 2002), Piedimonte Matese (CE) 2002, 302-316.

F. SIRANO, Identità culturali nella Campania settentrionale: un aggiornamento, in C. CORSI, E. POLITO (a cura di), Dalle sorgenti alla foce. 
Il bacino del Liri - Garigliano nell'antichità: culture contatti scambi, Atti del Convegno (Frosinone - Formia 2005), Roma 2008, 37-59.

P. TALAMO, L'area aurunca nel quadro dell'Italia centromeridionale. Testimonianze archeologiche di età arcaica (BAR Int.Ser. 384), Oxford 1987.

P. TALAMO, Nuove scoperte di età eneolitica e del bronzo nell'ambito della preistoria del territorio alifano, in F. MIELE, F. SIRANO (a cura di), Ager allifanus. La Piana Alifana alla Luce delle Recenti Ricerche Archeologiche, Catalogo della Mostra (Alife, Museo Archeologico, 29 maggio - 29 ottobre 2004), Quaderni Campano-Sannitici IV, Piedimonte Matese (CE) 2004, 33-45.

R.J.A. TAlBERT (ed.), Barrington Atlas of the Greek and Roman World, Oxford 2000.

G. TAgLiamonte, I Sanniti, Milano 1997.

G. TAgliamonte, L'età preromana e sannitica. Le necropoli preromane nell'ambito della cultura sannitica nel territorio alifano, in F. Miele, F. SirAno (a cura di), Ager allifanus. La Piana Alifana alla Luce delle Recenti Ricerche Archeologiche, Catalogo della Mostra (Alife, Museo Archeologico, 29 maggio - 29 ottobre 2004), Quaderni Campano-Sannitici IV, Piedimonte Matese (CE) 2004, 47-58.

G. Tagliamonte, I culti preromani, in S. CAPInI, P. CurCI, M.R. PICUTI (a cura di), Fana, templa, delubra. Corpus dei luoghi di culto dell'Italia antica, 3, Regio IV, Alife, Bojano, Sepino, Roma 2014, 11-14. P. ZANOVELlO, Le fonti epigrafiche, in I. RIERA (a cura di), Utilitas necessaria. Sistemi idraulici nell'Italia romana, Milano 1994, 99-143.

"Il rapporto uomo acqua nelle scelte insediative e nell'organizzazione..." 